\title{
Long-term correlations in solar proxies and solar wind parameters
}

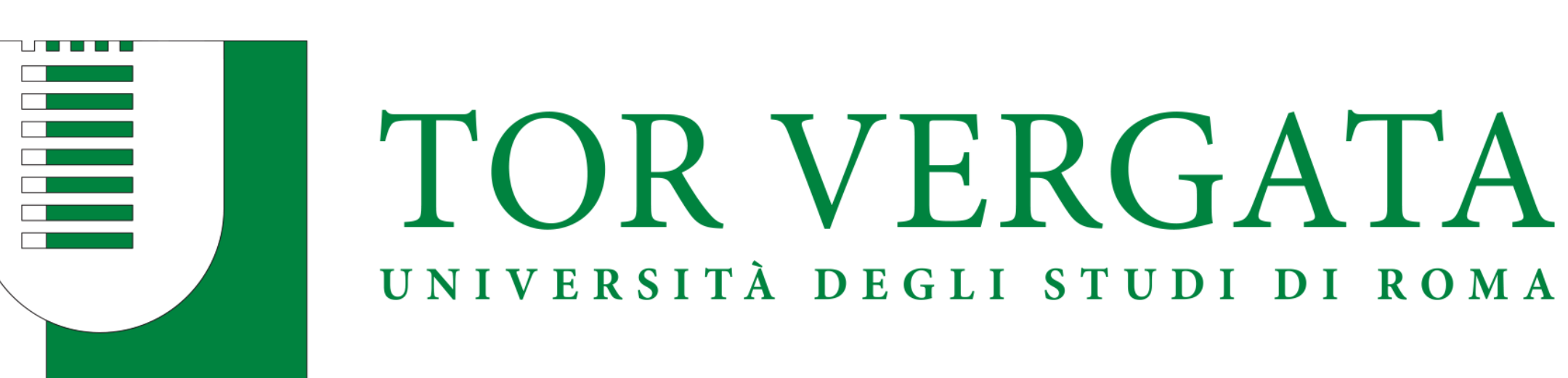 Uriv}

Abstract

Solar variability related to its magnetic activity can be quantified by using synthetic indices (e.g. sunspots number) or physical ones (e. chromospheric proxies). In order to connect the long-term solar activity variations to solar wind properties, we use Ca II K index and solar wind OMNI data in the time interval between 1965 and 2019, which almost entirely cover the last 5 solar cycles (20-24). A time lag in the correlation between the parameters is found. This time shift seems to show a temporal evolution over the different solar cycles.

The advantage to use a chromospheric proxy opens the possibility to extend the relation found for the Sun to link stellar variability and stellar wind properties in Sunlike stars.

\section{Introduction}

Manifestations of solar magnetic activity include several energetic phenomena (e.g. flares, CMEs, CIRs, solar wind) which span a wide range of time-scales. The solar wind constitutes an example of stationary phenomena. which strongly interacts with the Earth's magnetic field compressing and pertubing it, and providing, as a result of the interaction, the shape to the Earth's magnetosphere. For this and other reasons space climate analysis, which focus on the long-term variations of the solar activity and its near-Earth effects on scales from years to several millennia, constitute topic of paramount importance.

A possible relation between solar activity and solar wind parameters has been investigated starting from 1970s [1,2] showing the presence of a possible solar wind cycle not in phase with the solar one. Instead, the first direct evidence of changes in solar wind connected to changes on the Sun was reported by [3]. In a later analysis, a specific time lag between SSN and solar wind speed time series has been found [4].

In this work instead of the SSN, which strongly depends on the surface manifestation of concentrated magnetic structures, we use measures of a physical proxy of the solar activity, the Ca II K index, and solar wind OMN data to derive their long-term relations by mean of an appropriate filtering.
R. Reda ${ }^{(1,2)}$, T. Alberti (2), F. Berrilli ${ }^{(1)}$, P. Giobbi (1), L. Giovannelli (1), V. Penza ${ }^{(1)}$

(1) Deparment of Physics, University of Rome Tor Vergata, Via della Ricerca Scientifica 1, 00133, Rome, Italy

(2) INAF - Istituto di Astrofisica e Planetologia Spaziali, Via del Fosso del Cavaliere 100, 00133, Rome, Italy

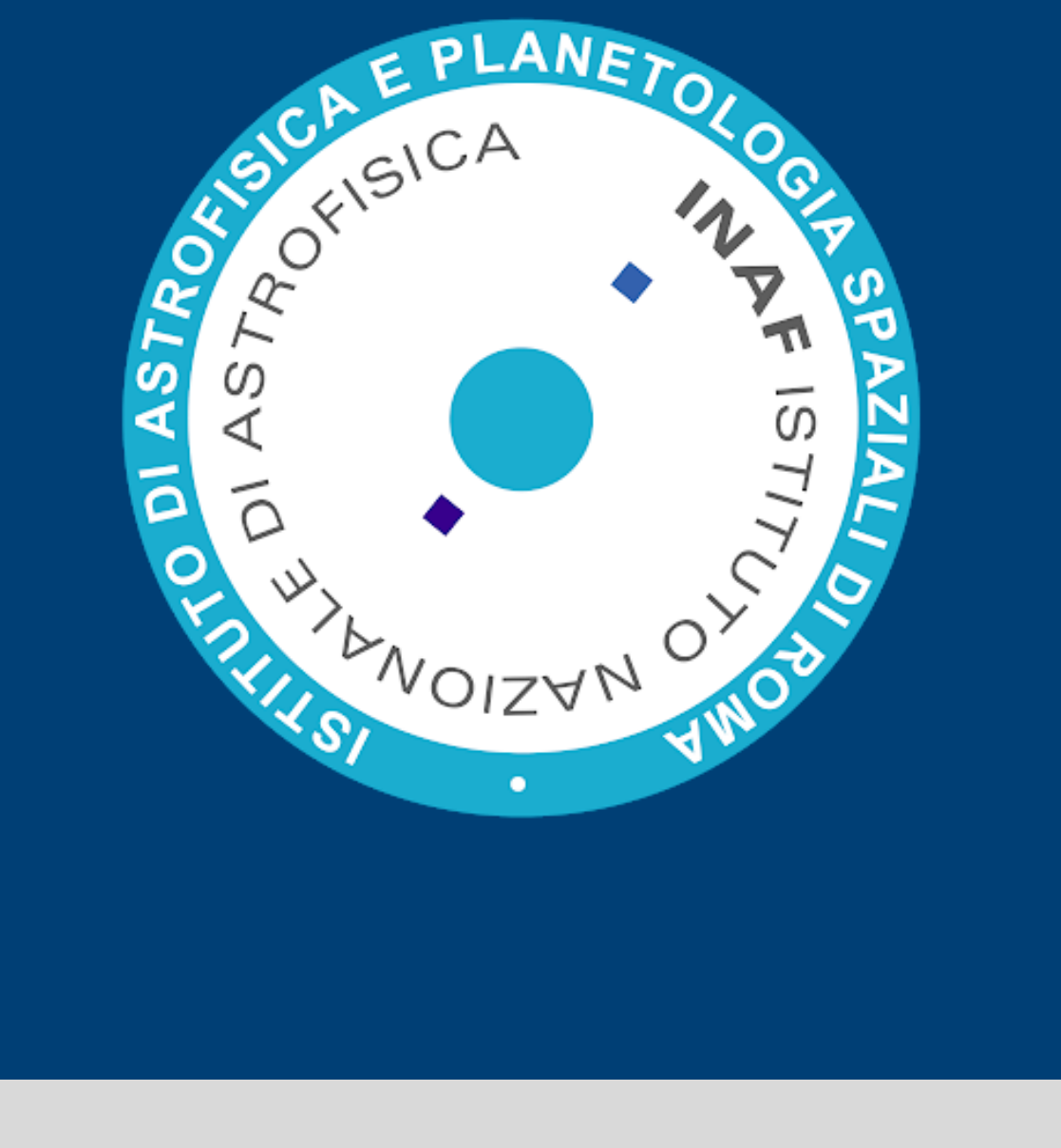

\section{Datasets}

The dataset used for this work is composed by: - Call K index (July 1965 - October 2017);

- Mg II index (November 1978 - December 2019);

Solar wind speed and dynamic pressure OMNI data (July 1965 - December 2019).

We use the Ca II K index time series from National Solar Observatory (NSO) [5], which gives monthly means up to October 2017. Then, due to the fact that the Ca II K index strongly correlate with the Mg II index we use the latter to extend $\mathrm{Ca} \| \mathrm{K}$ index until December 2019.

Solar wind parameters can be obtained by means of the OMNI database, which contains magnetic field and plasma measurements at different time resolutions. In particular, we consider plasma measurements of density $\mathrm{n}$ and speed $v$ at hourly resolution, from which we compute the solar wind dynamic pressure as $P=1 / 2$ $m_{p} n v^{2}$.

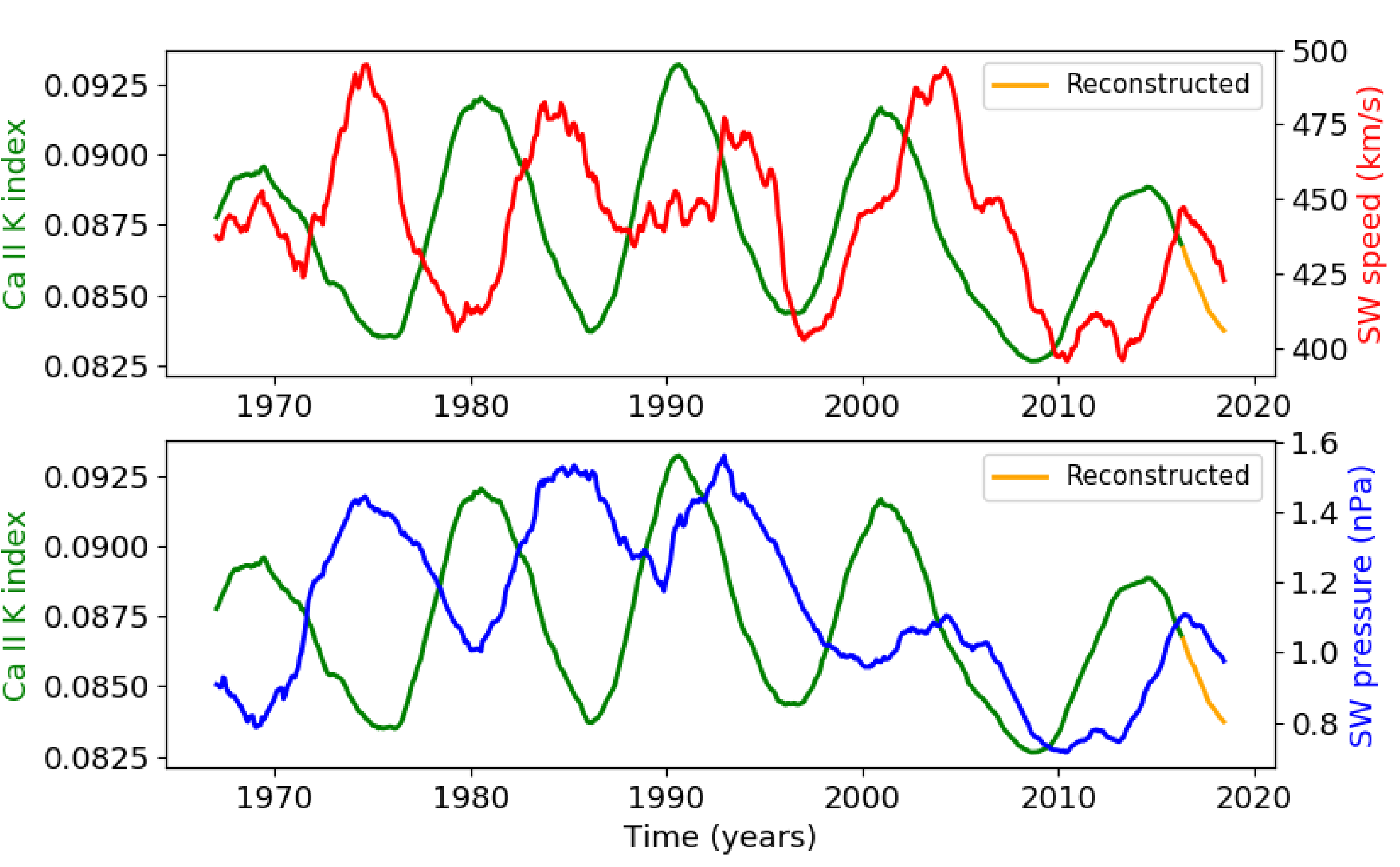

Therefore, our dataset consists of monthly means of the Ca II K index and two solar wind parameters, such as speed and dynamic pressure, in the time interval between July 1965 up to December 2019, which almost entirely covers the last 5 solar cycles (20-24).

To study their long-term relation, we apply a 37-month moving averages to our datasets, following a quite similar approach to that used by [4]. This choice is to remove any presence of variability with solar-rotation and yearly time-scales. The 37-month averaged data are shown in the above figures.

\section{Data analysis}

The 37-month averaged data of both SW speed and dynamic pressure show a cycle with a period quite similar to the 11-year solar cycle. Nevertheless, no clear correlation between solar wind speed (dynamic pressure) and Ca II K index seems to hold over the entire period $R=-0.05 \quad(R=-0.02)$. Instead, we found a strong anti-correlation for solar cycles 20 and 21, while this trend is not visible in the last 3 solar cycles [6]. This result, which is shown in the below plots, confirm the result recently reported by [7] on SSN and solar wind dynamic pressure.
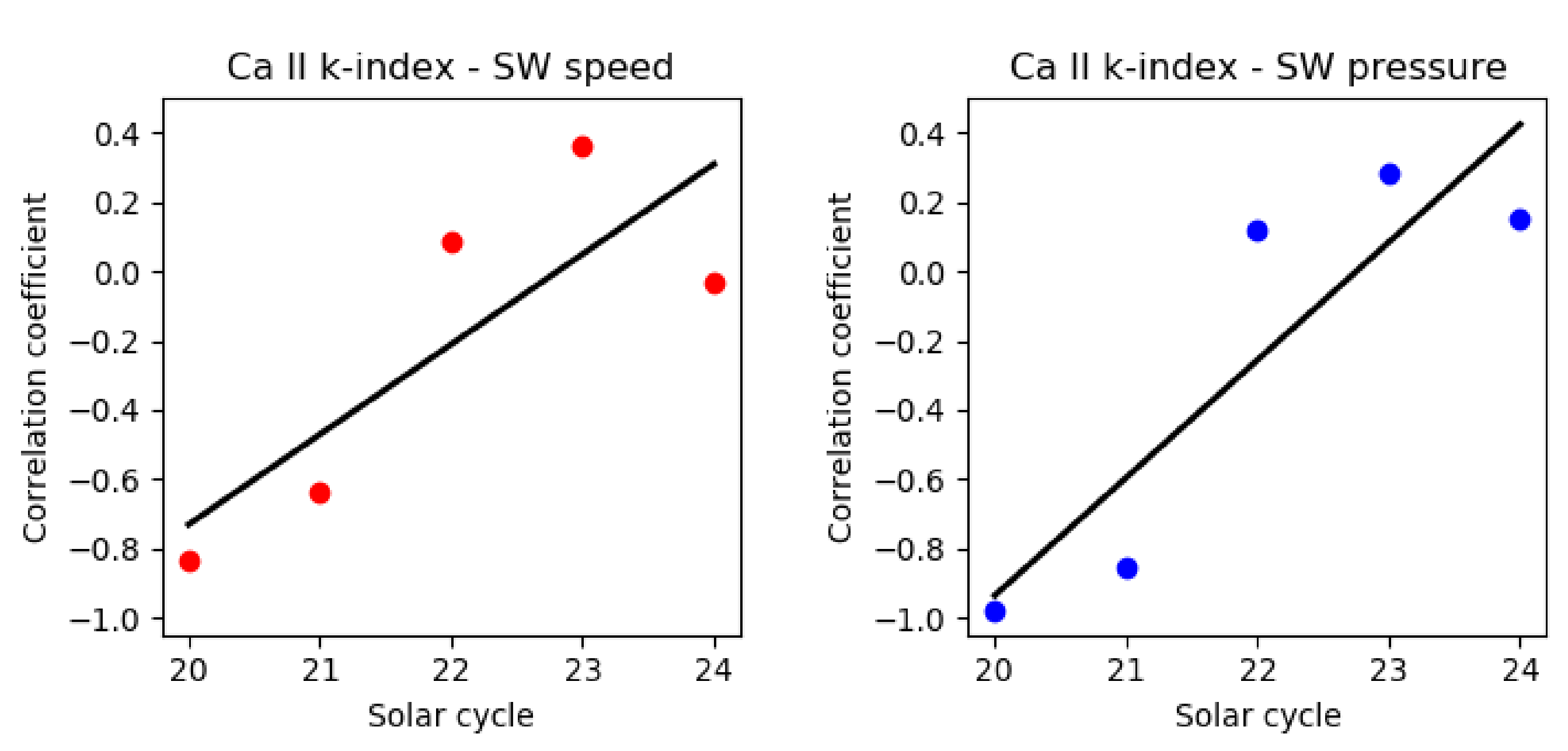

Computing a cross-correlation analysis, we found: - 3.2-year lag between Ca II K index and SW speed; 3.7-year lag for Ca II K index and SW dynamic pressure

Moreover, by computing the cross-correlation over single solar cycles, we found that those time shifts are not constant over the whole period, but show an interesting temporal evolution.

The time trend found for the lag of both solar wind parameters with respect to $\mathrm{Ca}$ II K index is a typical characteristic of time-series which show different periodicities, like the ones used for this work.

We then corrected our dataset for the different main periodicities of each time-series and finally we obtained a correlation relation which is valid for the entire time interval investigated.

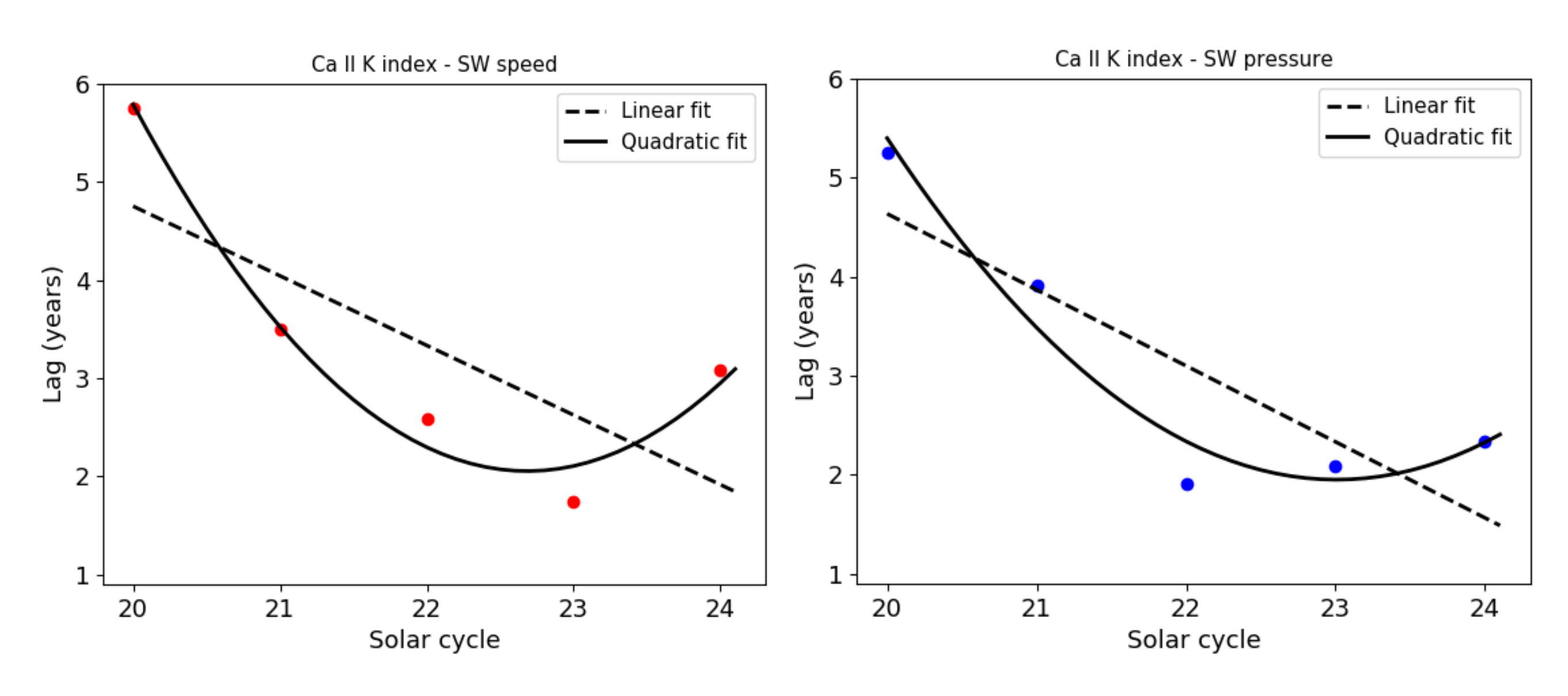

\section{Conclusions}

We analyzed the long-term behaviour of a solar activity proxy, the Ca II K index, and two solar wind parameters, such as speed and dynamic pressure, for the last five solar cycles.

What we found is:

- No clear correlation over the entire period

Anti-correlation for solar cycles 20 and 21 , while it moves toward a correlation for the last three cycles; 3.2 years lag between $\mathrm{Ca} I I \mathrm{~K}$ index and SW speed, while it is 3.7 for SW pressure. In both cases the lag is not constant over the time;

- Typical characteristic of time-series which show different dominant frequencies;

Correcting for them, a correlation relation valid for the entire period is found;

Because of the use of a chromospheric proxy, the relation found for the Sun can be easily extended to Sun-like stars to link stellar variability and stellar wind properties.
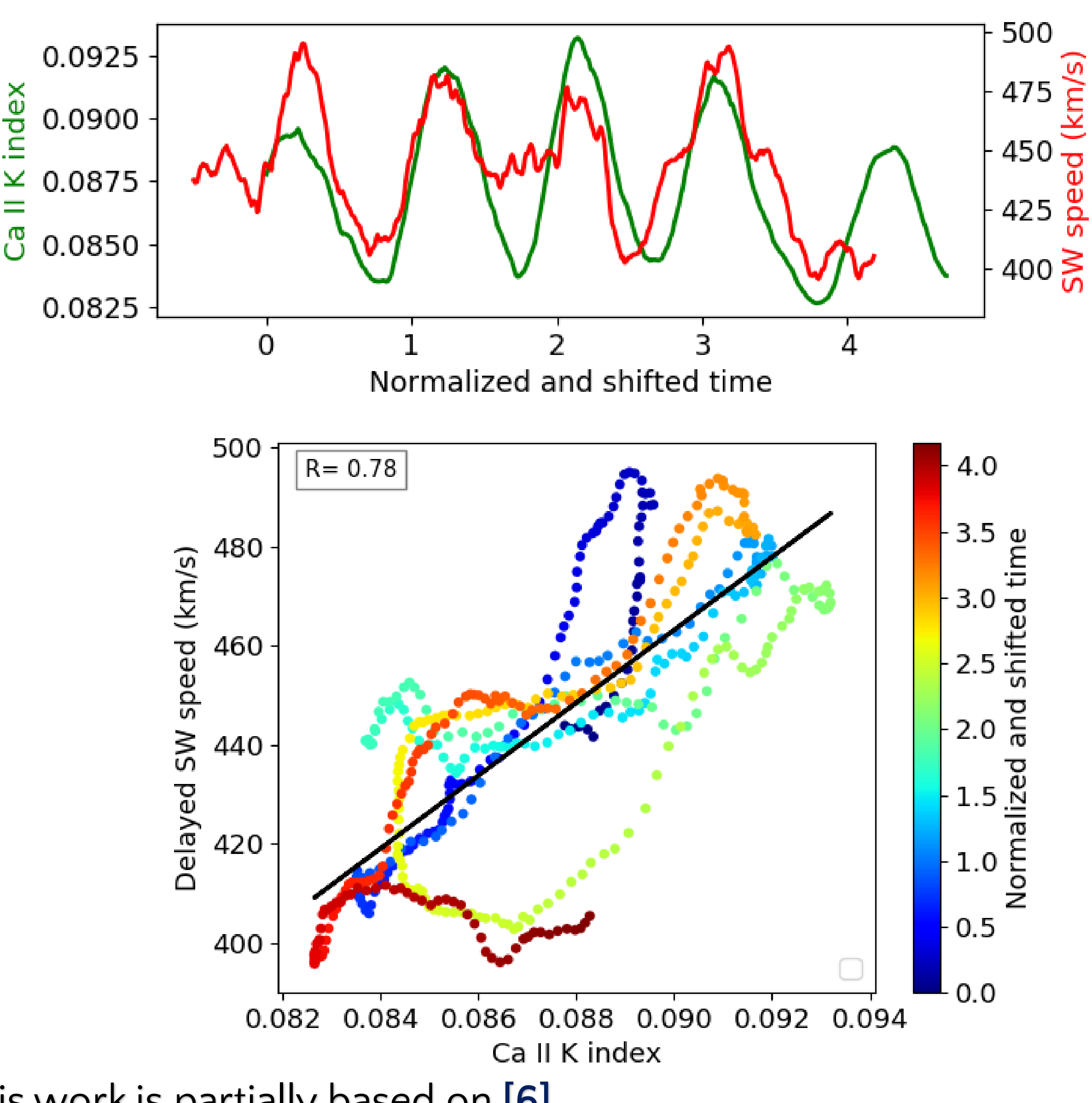

This work is partially based on [6].

References

[1] Hirshberg J., Astrophys. Space Sci. 20 (1973), 473-48 [2] Feynman J., Ceophys. Res., 87 (1982) A8, 6153-6 62 [4] Kohnlein W., Astrophys. Space Sci., 245 (1996), 81-88 [5] Bertello L. at al., Solar Phys., 291 (2016), 2967-2979 [6] Reda R. et al., Nuovo Cimento, (2021), submitted [7] Samsonov A. et al.. J. Geophys. Res., 124 (2019), 4049-4063 